\title{
The structure of the mental lexicon: what primary progressive aphasias reveal
}

Clara Sanches ${ }^{1}$, Alexandre Routier ${ }^{1,2,3}$, Olivier Colliot ${ }^{2,3,4}$, Marc Teichmann $^{1,5}$

${ }^{1}$ Sorbonne Universités, UPMC University Paris 6, INSERM, CNRS, Institut du cerveau et la moelle épinière (ICM), FrontLab Team, Hôpital Pitié-Salpêtrière, Paris, France

${ }^{2}$ Sorbonne Universités, UPMC University Paris 6, INSERM, CNRS, Institut du cerveau et la moelle épinière (ICM) - Hôpital Pitié-Salpêtrière, Paris, France

${ }^{3}$ Inria Paris, Aramis project-team, Paris, France

${ }^{4}$ Departments of Neuroradiology and Neurology, Hôpital Pitié-Salpêtrière, AP-HP, Paris, France.

${ }^{5}$ Department of Neurology, Institut de la mémoire et de la maladie d'Alzheimer, National Reference Center for « Rare Dementias ». Hôpital Pitié-Salpêtrière, AP-HP, Paris, France.

\section{Correspondence to:}

Marc Teichmann

Department of Neurology, National Reference Center for « Rare Dementias »

Hôpital de la Pitié Salpêtrière, 47-83, boulevard de l'Hôpital. 75013 Paris. France.

E-mail: marc.teichmann@psl.aphp.fr

Telephone: +331421675 34. Fax: +33142167504 


\section{Abstract}

Like recursive syntax, a structured mental lexicon is specific to the human species but its internal organization remains unclear. It is thought to contain information about the semantic, syntactic (e.g., gender) and formal (orthographic/phonological) features of a word. Previous studies suggested that these three components might be separated at the behavioral level and that they might be implemented by temporal cortices. However, the available investigations are based on case reports or small-cohort studies with patients demonstrating post-stroke aphasia, and they did not contrast the three lexical components in a directly comparable way. Similarly, functional imaging studies with healthy adults did not compare the lexical components but explored them separately using various tasks. Here we assessed the three components with comparable tasks in a relatively large cohort of 20 patients with primary progressive aphasia (PPA), namely logopenic and semantic PPA, which have been shown to affect the temporal cortex. The same tasks were also applied to 23 healthy adults. We thereby primarily aimed at showing multiple intra-lexical dissociations at the behavioral level to demonstrate the existence of a threefold segregation within the mental lexicon. We also sought to confirm the temporal-cortical involvement in the implementation of the lexical components and to characterize differential lexical breakdown in PPA. Lexical components were explored with three implicit processing tasks (semantic, syntactic-gender, word-form priming) and with three explicit matching tasks (semantic, syntactic-gender, word-form). Our results indicate that the three components are functionally segregated as evidenced by multiple dissociations at the group level, and the individual level, thus substantiating the existence of a threefold structure of the mental lexicon. Cortical thickness analyses showed damage to the left lateral temporal cortex in the entire PPA cohort suggesting that lexical components are anatomically segregated within this cortical region. Our results also refine 
previous proposals about lexical deficits in PPA by demonstrating differential damage to all three components of the lexicon in semantic and logopenic PPA, which might have an impact on PPA diagnosis and language rehabilitation strategies.

Keywords: lexicon, primary progressive aphasia, semantic/syntactic/word-form components, temporal cortex 


\section{Introduction}

The concept of the 'mental lexicon' was first introduced by Oldfield (1966) who suggested the existence of a 'mental dictionary' in which information about word meaning is retrieved. It is now suggested that the 'dictionary' should be regarded not only as a repertory of word meanings but as a three-fold lexicon comprising syntactic, semantic and word-form (phonological/orthographical) features (e.g., Jackendoff, 2002). However, there are distinct models regarding the organization of the lexicon. In the influential model of Levelt (1992) lexical access occurs serially along two stages: first, the selection of semantic and syntactic representations (lemma level) and, second, the selection of its phonological/orthographic content (lexeme level). Caramazza and Miozzo (1997) proposed an alternative model according to which semantic, syntactic and word-form features can be accessed independently. One argument for the latter model is provided by the « word-on-the-tip-of-thetongue » phenomenon demonstrating that access to semantic and syntactic information is independent from phonological or orthographic information (Caramazza and Miozzo, 1997). The authors have explored this phenomenon in individual cases analyzing the correlation between the retrieval of lexical-syntactic gender inforrmation, and of word-form information such as the first phoneme of words that subjects could not retrieve. The absence of significant correlations indicated that access to word-form information is independent from the access to syntactic, and probably semantic, information. Using lesion models, Kavé and Levy (2003) also provided support for separate lexical components by showing that Alzheimer's disease patients can have intact access to syntactic information like noun gender despite deficits in accessing semantic information. The opposite pattern has been evidenced in a small cohort of post-stroke patients suggesting that lexical-syntactic information, namely gender and predicate argument structure, might be stored separately from lexical-semantics (Biran and 
Friedmann, 2012). In summary, the available investigations suggest that lexical structure probably has different levels which are independent but connected: semantic, syntactic and word-form representations. However, even if the aforementioned case reports and smallcohort studies have suggested functional dissociations between the lexical components, evidence for an intra-lexical segregation can not be generalized without exploring homogenous and large patient cohorts. Furthermore, none of the previous studies has provided direct comparisons between the three lexical components, thus indicating the need for directly comparable tasks to substantiate the three-component hypothesis of the lexicon.

A related issue is the anatomical segregation of the lexical components. Most imaging studies have suggested that the lateral temporal cortex might be involved in the processing of lexical retrieval, but there is no absolute consensus. Functional magnetic resonance imaging (fMRI) with healthy adults exploring lexical-semantic processing have shown activation in various brain areas including the middle temporal and the superior temporal gyrus, the anterior temporal lobe, the inferior frontal gyrus and/or the inferior parietal lobe (e.g., Kuperberg et al., 2008; Lau et al., 2013; Ruff et al., 2008). Using lesion models, particularly the semantic variant of primary progressive aphasia (sv-PPA), which is characterized by degenerative damage to the anterior temporal lobe and semantic deficits, have reinforced the claim about a potential role of this region in lexical semantics (Mesulam et al., 2013; Wilson et al., 2014). Other researchers, however, attribute pivotal roles for lexical-semantics to left prefrontal and temporal-parietal regions (Binder et al., 2003; Devlin et al., 2000; Garavan et al., 2000; Spitsyna et al., 2006). The anatomical correlate for the processing of word-form information is also still debated. Studies using fMRI in healthy adults showed that posterior and middle regions of the temporal lobe and/or the left supramarginal and the fusiform gyrus might be involved in mapping word-orthography on word-phonology or when accessing whole-word forms (e.g., Graves et al., 2008; 2010). Regarding the rare studies exploring syntactic word 
information, Snjiders et al. (2009) have suggested that left inferior frontal gyrus contributes to syntactic unification whereas the left posterior temporal gyrus subtends the retrieval of lexical-syntactic information. Taken together, previous studies suggest that, among other regions, the lateral temporal cortex might play a central role in lexical processing, but none of them has directly contrasted the three lexical components via comparable tasks. Thus, strong evidence for the anatomical implementation of the potentially distinct lexical components in temporal-cortical regions remains to be provided.

Here we addressed these issues by using PPA variants as a lesion model of the lateral temporal cortex and by applying directly comparable tasks tapping semantic, syntactic and word-form information, with the aim of providing robust evidence for multiple dissociations between the three lexical components. Based on the data-driven hypothesis that the left temporal cortex might play a central role in lexical processing, we explored two PPA variants which have been shown to affect this region: logopenic-variant PPA (lv-PPA) which usually damages posterior and middle portions of temporal cortices (besides the posterior-inferior parietal cortex), and sv-PPA demonstrating atrophy of anterior and middle portions of lateral temporal cortices (Gorno-Tempini et al., 2004). PPA is a neurodegenerative condition subdivided into three main variants: sv-PPA characterized by multi-modal semantic deficits, lv-PPA characterized by word-finding problems and verbal short-term memory failure, and agrammatic/nonfluent PPA which does not directly affect lexical processing but rather combinatorial language aspects such as word sequencing through syntax (Gorno-Tempini et al., 2011; Mesulam et al., 2014). As the aim of the present study was to explore lexicalized aspects of syntax we did not include this latter PPA variant in the investigation. This noninclusion of agrammatic/nonfluent PPA is also in line with evidence that lexical aspects of syntax appear to be implemented by posterior temporal regions (Snjiders et al., 2009) and not by Broca's area which subtends combinatorial syntax (e.g., Friederici et al., 2003; Pallier et 
al., 2011), and which is damaged in the agrammatic/nonfluent variant (Gorno-Tempini et al., 2004). Using sv-PPA and lv-PPA as a combined lesion model of temporal cortices also has an advantage over investigations of post-stroke cohorts because regions that are normally spared by stroke such as the anterior temporal cortex (Caviness et al., 2002; Wise, 2003) are affected in PPA. The use of PPA in this study also allows for addressing a related issue in that it enables a fine-grained characterization of lexical deficits in lv-PPA and sv-PPA.

\section{Methods}

\subsection{Participants}

Twenty PPA patients were recruited at the National Reference Center for "PPA and Rare Dementias" of the Pitié Salpêtrière Hospital (Paris): nine patients with sv-PPA and eleven with lv-PPA. The diagnosis was established by expert neurologists following the international diagnostic criteria of Gorno-Tempini et al. (2011). Patients did not present neurological or psychiatric diseases other than PPA. Twenty-three healthy controls, matched with the patients for age $(t(41)=-1.332, p=0.19)$, level of education $(t(41)=-1.36, p=0.18)$ and gender $(p=0.76$; Fisher's exact test) were also included. Healthy controls were tested with the Mini Mental State Examination (MMSE; Folstein et al., 1975) to ensure the preservation of their global cognitive abilities (mean score 29.1/30 \pm 1.2 ; Normal $>26 / 30$ ). They did not have any neurological disease or physical problem that could interfere with cognitive functioning. All participants were native French speakers. Written consent was obtained from all participants and the study has been approved by the local ethical committee. Demographic data are illustrated in Table 1. 
Table 1 Demographic data of healthy controls, sv-PPA and lv-PPA patients

\begin{tabular}{lccc}
\hline & $\begin{array}{c}\text { Controls } \\
(\text { means } \pm \text { SD) }\end{array}$ & $\begin{array}{c}\text { sv-PPA } \\
(\text { means } \pm \text { SD) }\end{array}$ & $\begin{array}{c}\text { Iv-PPA } \\
(\text { means } \pm \text { SD) }\end{array}$ \\
\hline Number of subjects & 23 & 9 & 11 \\
Sex (women, men) & $10 \mathrm{~W} / 13 \mathrm{M}$ & $6 \mathrm{~W} / 3 \mathrm{M}$ & $4 \mathrm{~W} / 7 \mathrm{M}$ \\
Age (years) & $66.91 \pm 9.08$ & $70.44 \pm 6.06$ & $70.64 \pm 7.51$ \\
Handedness & $21 \mathrm{R} / 2 \mathrm{~L}$ & $8 \mathrm{R} / 1 \mathrm{~L}$ & $11 \mathrm{R}$ \\
Years of education & $14.8 \pm 3.4$ & $14.9 \pm 4.3$ & $15.3 \pm 2.7$ \\
Symptom duration (years) & $/ / / / / /$ & $3.2 \pm 1.1$ & $3.1 \pm 1.5$ \\
\hline
\end{tabular}

$\mathrm{SD}=$ standard deviation

\subsection{General cognitive/language assessment}

The general cognitive assessment included the MMSE and the Frontal Assessment Battery (FAB; Dubois et al., 2000). The language assessment was composed of a picture naming test (D080; Deloche and Hannequin, 1997) and the Boston Diagnostic Aphasia Evaluation (BDAE; Mazaux and Orgogozo, 1982). The BDAE included an evaluation of aphasia severity taking into account spontaneous speech and the description of the 'cookies theft picture', a sentence repetition task, a single-word comprehension task requiring pointing to pictures upon auditory word presentation, and a verbal fluency test comprising phonemic and category fluency (Cardebat et al., 1990). According to the international diagnostic criteria of GornoTempini et al. (2011) all sv-PPA patients had single-word comprehension deficits and anomia (without sentence repetition disorders, agrammatism or motor speech disorders), and lv-PPA patients were characterized by word finding difficulties and sentence repetition impairment 
(without agrammatism, single-word comprehension or motor speech disorders).

Cognitive/language scores are summarized in Table 2.

Table 2 Cognitive/language scores of sv-PPA and lv-PPA patients

\begin{tabular}{lccc}
\hline & $\begin{array}{c}\text { Sv-PPA } \\
(\text { means } \pm \text { SD) }\end{array}$ & $\begin{array}{c}\text { Iv-PPA } \\
(\text { means } \pm \text { SD) }\end{array}$ & $\begin{array}{c}\text { Normal } \\
\text { threshold }\end{array}$ \\
\hline MMSE & $24.8 \pm 3.1$ & $21.6 \pm 3.1$ & $\geq 27$ \\
FAB & $14.6 \pm 2.6$ & $13.9 \pm 3.1$ & $\geq 16$ \\
BDAE - aphasia severity scale & $3.8 \pm 1.0$ & $3.4 \pm 1.1$ & $>4$ \\
BDAE - single-word comprehension & $59.5 \pm 4.4$ & $69.5 \pm 3.6 *$ & $\geq 68$ \\
BDAE - sentence repetition & $14.9 \pm 3.2$ & $10.5 \pm 3.1 *$ & $\geq 14$ \\
Category fluency & $10.0 \pm 6.1$ & $15.6 \pm 4.7 *$ & $\geq 15$ \\
Phonemic fluency & $13.1 \pm 8.4$ & $12.9 \pm 7.5$ & $\geq 15$ \\
DO80 & $39.7 \pm 16.7$ & $59.2 \pm 15.4 *$ & $\geq 75$ \\
\hline
\end{tabular}

$\mathrm{SD}=$ standard deviation. $\mathrm{MMSE}=$ Mini Mental State Examination. FAB $=$ Frontal Assessment Battery. BDAE $=$ Boston Diagnostic Aphasia Examination. DO80 $=$ Picture naming task. Asterisks indicate significant differences between sv-PPA and lv-PPA.

\subsection{Experimental tasks}

\subsubsection{Implicit processing tasks}

The three implicit processing tasks used priming paradigms tapping semantic, syntactic and word-form information. Priming reflects shorter reaction times making judgments about target words that are preceded by related prime words as compared to target words preceded by unrelated primes (Neely, 1976; Kuperberg et al., 2008). A task frequently used in priming studies is 'lexical decision' where real words and non-words are used as targets and subjects 
have to decide whether the item exists or not in their mother tongue (e.g., Kuperberg et al., 2008).

In the semantic task, we used 45 pairs with $2 / 3$ of real words as targets and $1 / 3$ of non-words. The primes were either semantically associated with the target or without any semantic relationship (e.g. honey-bee vs. cardboard-bee). In the word-form task we used 45 pairs with $2 / 3$ of real words as targets and $1 / 3$ of non-words. The primes were words with a similar spelling code or with no similarity (e.g. deer-beer $v s$. foot-beer). In the syntactic task, we focused in noun gender information using 52 pairs with $2 / 3$ of real words as targets (inanimate items for which no natural gender could be inferred) and 1/3 of non-words. The primes were determiners that either agreed in gender with the target or not. French language was used across all tasks given that French, unlike English, provides the opportunity to test syntactic gender. Each priming task contained two lists of prime-target pairs. In each list half of the targets were paired with related primes and the other half with unrelated primes. This was done to avoid a target appearing twice in a list, which could lead to biasing repetition priming effects. Related and unrelated primes were matched for frequency and number of letters (all Fs $<1$ ) using the LEXIQUE 2 database (New et al., 2004). All non-word targets were orthographically and phonotactically legal and had the same number of letters as target words (all Fs $<1$ ). Figure 1A illustrates the three tasks.

They were presented on a computer using the E-prime software and were applied at two different moments of the day (morning/afternoon, list 1/list 2) in order to minimize eventual repetition priming effects for a given target. Half of the participants saw one of the two lists in the morning and the other in the afternoon, and the other half of the participants saw them in the reverse order. For the semantic and syntactic tasks, each trial began with a fixation cross (800ms), followed by the prime word written in black $(150 \mathrm{~ms})$, then a blank screen $(50 \mathrm{~ms})$, followed by the target written in red $(4 \mathrm{~s} ; \mathrm{SOA}=200 \mathrm{~ms})$. For the word-form task we used a 
masked priming paradigm. Each trial began with a fixation cross $(800 \mathrm{~ms})$ followed by a series of pound signs $(500 \mathrm{~ms})$. The prime was then presented in lowercase characters $(67 \mathrm{~ms})$, immediately followed by the target presented in capital letters to avoid purely visual effects $(4 \mathrm{~s} ; \mathrm{SOA}=67 \mathrm{~ms})$. This masked priming procedure was used because previous studies have shown that with unmasked conditions word-form priming effects generally do not arise (e.g. Velan and Frost, 2011). Between each trial there was a blank screen during 700ms.

The instructions given to the participants were to "be focused on the screen during the whole task without speaking", to "answer as fast and accurately as possible" and "to press the 'yes' button on the computer keyboard if the word target exists in French and the "no" button if the word doesn't exist". Participants answered by pressing "yes" and "no" with the index finger and with the middle finger of their dominant hand, respectively. Before each task participants were familiarized with the procedure by five training trials. The order of the three tasks was randomized.

\subsubsection{Explicit processing tasks}

Three additional explicit processing tasks were applied to screen for 'good' and 'poor' performers in the semantic, syntactic and word-form domains of the lexicon. They explicitly directed the participants' attention to associations between stimuli by asking them to make a judgment of links between the stimuli presented, thus involving conscious control processes (Kuperberg et al., 2008).

Thirty target words were selected using the Lexique 2 database (New et al., 2004). All targets were concrete French nouns. Half had feminine gender and half masculine gender, with word frequencies ranging from 570.3 to 0.67 per million. In the semantic task, each target was paired with two items, one being semantically related, either at a categorical level $(50 \%$ of stimuli) or at an associative level (50\% of stimuli), and a distractor without any semantic 
relation to the target. The targets, the related items and the distractors were matched for frequency $($ all Fs $<1$ ) and for the number of letters (all Fs $<1$ ). In the word-form task a letter was removed from each target, either the first or the last. Two letters were presented along with the target, one that completed the word and a distractor letter that did not allow for providing a real French word although it allowed for an orthographically legal word. In the syntactic task two determiners were presented along with the target. One determiner agreed in gender with the target whereas the distractor did not. All targets were presented at the top of the computer screen and were accompanied by the related item and the distractor at the left or right bottom of the screen. In half of the trials the distractor was on the left side, in the other half it was on the right. The noun stimuli used in the explicit processing tasks were all distinct from the words of the priming tasks. Figure 1B illustrates the three explicit tasks.

They were conducted after the application of the first three lists of the priming tasks. Stimuli were presented on a computer screen. The word-form task was always presented first so that subjects did not see the full word before this word completion task. The order of the two other tasks was randomized. In the semantic task the participants were instructed to point with the index finger of the dominant hand to the word that was associated with the target word. In the word-form task the instruction was to point to the letter that completed the word, and in the syntactic task participants were required to point to the determiner that was in gender agreement with the target. Each task was preceded by five training trials to familiarize the participants with the tasks.

\subsection{Data analyses, MRI acquisition and image processing}

For the priming tasks, analyses (ANOVAs) compared reaction times (RT) for related and unrelated targets. Priming effects were defined by the RT contrast between unrelated minus related prime-target pairs. For the explicit tasks the analyses were based on performance 
accuracy. To check for dissociations between the three lexical components, the whole PPA group was subdivided into two subgroups according to the performance on the explicit tasks. This subdivision resulted in subgroups of "good performers" (SEM+ $[\mathrm{N}=10], \mathrm{SYNT}+$ $[\mathrm{N}=10]$, FORM $+[\mathrm{N}=10])$, and of "poor performers (SEM- $[\mathrm{N}=10]$, SYNT- $[\mathrm{N}=10]$, FORM$[\mathrm{N}=10])$. Priming effects were then reanalyzed for these subgroups using ANOVAs.

High resolution T1-weighted MRIs were acquired at the Pitié-Salpêtrière Hospital for 17 out of 20 PPA patients and for 12 healthy controls using a magnetization-prepared rapid gradient echo sequence (MPRAGE). Cortical thickness measurements were performed from T1weighted images using FreeSurfer 5.3 software (http://surfer.nmr.mgh.harvard.edu/). Briefly, image processing included removal of non-brain tissue using a hybrid watershed/surface deformation procedure, automated Talairach transformation, intensity normalization, tessellation of the grey matter-white matter boundary, automated topology correction and surface deformation following intensity gradients to optimally place the grey matter-white matter and grey matter-cerebrospinal fluid borders at the location where the greatest shift in intensity defined the transition to the other tissue class. Cortical thickness was calculated by measuring the distance between representations of the grey matter-white matter and grey matter-cerebrospinal fluid boundaries across each vertex of the tessellated surface (Fischl and Dale, 2000). All cortical thickness maps were registered onto the common template provided with FreeSurfer (FSAverage template).

Surface-based analyses of cortical thickness were performed using SurfStat software (http://www.math.mcgill.ca/keith/surfstat/). Cortical thickness maps were smoothed using a $20 \mathrm{~mm}$ surface-based kernel. The comparison of cortical thickness between groups was carried out with a two-sample t-test at each vertex (sv-PPA vs. controls; lv-PPA vs. controls; all PPA patients vs. controls). In all analyses, statistics were corrected for multiple comparisons using the random field theory for non-isotropic images (Worsley et al., 1999). A 
statistical threshold of $\mathrm{p}<0.001$ was first applied. An extent threshold of $\mathrm{p}<0.05$ corrected for multiple comparisons was then applied at the cluster level.

\section{Results}

\subsection{Implicit processing tasks}

Regarding performance accuracy, PPA patients had slightly lower performance $(94.2 \%$ correct \pm 6.1 ; lv-PPA $90.6 \%$ correct \pm 9.4 , sv-PPA $97.8 \%$ correct \pm 2.8$)$ than controls $(99.5$ $\%$ correct \pm 0.9 ), but the overall high accuracy percentage in PPA patients showed that they reliably and accurately performed the lexical decision task.

ANOVAs were conducted by participants (F1) and by items (F2) with reaction times (RT) as the dependent variable. For each participant, incorrect responses and missing data were excluded from the analyses. The independent variables were "group" (PPA, controls) and "target type" (related, unrelated). Analyses were conducted separately on the three priming tasks given that they slightly differed in the experimental design (masked word-form priming, unmasked semantic and syntactic priming). Figure 2 summarises the RT with the tasks for controls and PPA patients.

In the semantic priming task RT were slower in PPA (1144 ms \pm 386$)$ than in controls (791 $\mathrm{ms} \pm 228 ; \mathrm{F} 1(1,41)=14.04, \mathrm{p}=0.001 ; \mathrm{F} 2(1,58)=396.14, \mathrm{p}<0.001)$. There was a target type effect (related $921 \mathrm{~ms} \pm 365$, unrelated $989 \mathrm{~ms} \pm 349 ; \mathrm{F} 1(1,41)=17.53, \mathrm{p}<0.001$; $\mathrm{F} 2(1,58)=8.00, \mathrm{p}=0.006)$ and a target type $\times$ group interaction $(\mathrm{F} 1(1,41)=6.33, \mathrm{p}=0.016$; $\mathrm{F} 2(1,58)=6.93, \mathrm{p}=0.011)$. The interaction was linked to the fact that controls had faster RT with related targets $(738 \mathrm{~ms} \pm 203)$ than with unrelated targets (844 ms \pm 244 ; $\mathrm{F} 1(1,22)=25.40, \mathrm{p}<0.001 ; \mathrm{F} 2(1,58)=34.23, \mathrm{p}<0.001)$ whereas there was no difference in PPA (related $1132 \mathrm{~ms} \pm 400$, unrelated $1156 \mathrm{~ms} \pm 382$; both $\mathrm{Fs}_{\mathrm{s}}<1$ ). The magnitude of the significant priming effect in controls was $106 \mathrm{~ms}$ whereas in PPA patients the non significant 
effect was $24 \mathrm{~ms}$. Restricted analyses compared RT in lv-PPA and sv-PPA for related vs. unrelated targets. Both PPA groups had similar RT (lv-PPA $1151 \mathrm{~ms} \pm 447$; sv-PPA $1136 \mathrm{~ms}$ \pm 307 ; both Fs $<1$ ). There was no effect of target type (related lv-PPA $1128 \mathrm{~ms} \pm 465$, related sv-PPA $1137 \mathrm{~ms} \pm$ 331; unrelated lv-PPA $1173 \mathrm{~ms} \pm$ 451, unrelated sv-PPA $1135 \mathrm{~ms} \pm$ 301; both Fs $<1$ ), and no group $\mathrm{x}$ target type interaction (both $\mathrm{Fs}<1$ ).

In the syntactic priming task RT were slower in PPA $(1103 \mathrm{~ms} \pm 391)$ than in controls (781 $\mathrm{ms} \pm 207)(\mathrm{F} 1(1,41)=11.85, \mathrm{p}=0.001 ; \mathrm{F} 2(1,58)=112.24, \mathrm{p}<0.001)$. There was a target type effect (related $919 \mathrm{~ms} \pm 349$, unrelated $942 \mathrm{~ms} \pm 345 ; \mathrm{F} 1(1,41)=6.44, \mathrm{p}=0.011$ $\mathrm{F} 2(1,58)=6.11, \mathrm{p}=0.019)$ and a target type $\times$ group interaction $(\mathrm{F} 1(1,41)=5.24, \mathrm{p}=0.027$; $\mathrm{F} 2(1,58)=6.00, \mathrm{p}=0.023)$. The interaction was linked to the fact that controls had faster RT with related targets $(754 \mathrm{~ms} \pm 189)$ than with unrelated targets $(807 \mathrm{~ms} \pm 225 ; \mathrm{F} 1(1,22)=9.38$, $\mathrm{p}=0.006 ; \mathrm{F} 2(1,58)=17.99 \mathrm{p}<0.001)$ whereas there was no difference in PPA (related $1097 \mathrm{~ms}$ \pm 396, unrelated $1117 \pm 396$; both $\mathrm{Fs}<1$ ). The magnitude of the significant priming effect in controls was $53 \mathrm{~ms}$ whereas in PPA patients the non significant effect was only $20 \mathrm{~ms}$. Restricted analyses compared RT in lv-PPA and sv-PPA for related vs. unrelated targets. Both PPA groups had similar RT (lv-PPA $1072 \mathrm{~ms} \pm 422$; sv-PPA $1141 \mathrm{~ms} \pm 358$; both Fs $<1$ ). There was no effect of target type (related lv-PPA $1087 \mathrm{~ms} \pm 443$, related sv-PPA $1137 \mathrm{~ms} \pm$ 355; unrelated lv-PPA $1057 \mathrm{~ms} \pm$ 421, unrelated sv-PPA $1145 \mathrm{~ms} \pm 382$; both Fs $<1$ ), and no group $\mathrm{x}$ target type interaction (both $\mathrm{Fs}<1$ ).

In the word-form priming task RT were slower in PPA $(1239 \mathrm{~ms} \pm 333)$ than in controls $(859$ $\mathrm{ms} \pm 185)(\mathrm{F} 1(1,41)=22.71, \mathrm{p}<0.001 ; \mathrm{F} 2(1,58)=265.28, \mathrm{p}<0.001)$. There was a target type effect (related $1009 \mathrm{~ms} \pm 312$, unrelated $1061 \mathrm{~ms} \pm 338 ; \mathrm{F} 1(1,41)=10.50, \mathrm{p}=0.002$; $\mathrm{F} 2(1,58)=6.23, \mathrm{p}=0.015)$ and a target type $\times$ group interaction $(\mathrm{F} 1(1,41)=6.33, \mathrm{p}=0.021$; $\mathrm{F} 2(1,58)=5.93, \mathrm{p}=0.031)$. The interaction was linked to the fact that controls had faster RT with related targets $(823 \mathrm{~ms} \pm 154)$ than with unrelated targets (894 ms \pm 209 ; 
$\mathrm{F} 1(1,22)=16.04, \mathrm{p}=0.001 ; \mathrm{F} 2(1,58)=20.70, \mathrm{p}<0.001)$ whereas there was no difference in PPA (related $1224 \mathrm{~ms} \pm 312$, unrelated $1254 \mathrm{~ms} \pm 360 ; \mathrm{F} 1(1,19)=1.15, \mathrm{p}=0.296 ; \mathrm{F} 2(1,58)=1.51$ $\mathrm{p}=0.225$ ). The magnitude of the significant priming effect in controls was $71 \mathrm{~ms}$ whereas in PPA patients the non significant effect was only $30 \mathrm{~ms}$. Restricted analyses compared RT in lv-PPA and sv-PPA for related vs. unrelated targets. Both PPA groups had similar RT (lvPPA $1246 \mathrm{~ms} \pm 351$; sv-PPA $1230 \mathrm{~ms} \pm 319$; both Fs $<1$ ). There was no effect of target type (related lv-PPA $1244 \mathrm{~ms} \pm 335$, related sv-PPA $1200 \mathrm{~ms} \pm 299$; unrelated lv-PPA $1249 \mathrm{~ms} \pm$ 382, unrelated sv-PPA $1259 \mathrm{~ms} \pm 354 ; \mathrm{F} 1<1 ; \mathrm{F} 2(1,58)=1.65, \mathrm{p}=0.205)$, and no group $\mathrm{x}$ target type interaction (both $\mathrm{Fs}<1$ ).

In summary, controls demonstrated significant priming effects in the three priming tasks whereas PPA as a whole group, as well as lv-PPA and sv-PPA separately, did not display priming effects in the three tasks.

\subsection{Explicit processing tasks}

ANOVAs were conducted by participants but not by items given that the stimulus-items used in the three tasks were distinct. Performance accuracy was the dependent variable. The independent variables were "group" (PPA, controls) and "task" (semantic, syntactic, wordform). Figure 3 summarises the results.

Performance was poorer in PPA patients $(91.6 \%$ correct \pm 10.7$)$ than in controls $(99.4 \%$ correct $\pm 1.3 ; \mathrm{F}(1,41)=21.75, \mathrm{p}<0.001)$. There was a task effect (semantic task $95.0 \%$ correct \pm 9.2, syntactic task $98.7 \%$ correct \pm 3.9 , word-form task $93.6 \%$ correct \pm 9.9 ; $\mathrm{F}(2,82)=11.74, \mathrm{p}<0.001)$ and a group $\mathrm{x}$ task interaction $(\mathrm{F}(2,82)=9.64, \mathrm{p}<0.001)$. The interaction was linked to the fact that PPA patients had distinct performance for the three tasks (semantic task $90.0 \%$ correct \pm 11.7 , syntactic task $97.3 \%$ correct \pm 5.4 , word-form task $87.4 \%$ correct $\pm 11.6 ; \mathrm{F}(2,38)=10.21, \mathrm{p}<0.001)$ whereas controls had similar 
performance (semantic task $99.4 \%$ correct \pm 1.3 , syntactic task $99.9 \%$ correct \pm 0.7 , wordform task $99.0 \%$ correct $\pm 1.6 ; \mathrm{F}(2,44)=2.32, \mathrm{p}=0.110)$. Restricted analyses showed that PPA patients had lower performance for the three tasks (semantic task $F(1,41)=14.89, p<0.001$, syntactic task $\mathrm{F}(1,41)=5.01, \mathrm{p}=0.031$, word-form task $\mathrm{F}(1,41)=22.64, \mathrm{p}<0.001)$.

A second series of analyses compared performance between each of the three tasks in lv-PPA and sv-PPA to provide the performance pattern of the two PPA variants with respect to the three lexical components. Lv-PPA patients had lower performance in the word-form task $(85.9 \%$ correct \pm 13.2$)$ than in the semantic task $(93.0 \%$ correct $\pm 7.4 ; \mathrm{F}(1,10)=8.08$, $\mathrm{p}=0.017)$, and lower performance in the word-form task than in the syntactic task $(96.4 \%$ correct $\pm 6.1 ; \mathrm{F}(1,10)=9.40, \mathrm{p}=0.012)$. Performance was similar in the semantic and the syntactic task $(\mathrm{F}(1,10)=3.67, \mathrm{p}=0.085)$. Sv-PPA patients had similar performance in the wordform task $(89.2 \%$ correct \pm 9.8$)$ and the semantic task $(86.3 \%$ correct $\pm 15.0 ; \mathrm{F}<1$. They had lower performance in the semantic than in the syntactic task $(98.5 \%$ correct \pm 4.14 ; $\mathrm{F}(1,8)=8.49, \mathrm{p}=0.019)$, and in the word-form task than in the syntactic task $(\mathrm{F}(1,8)=10.33$, $\mathrm{p}=0.012$ ). Comparisons of performance between lv-PPA and sv-PPA versus controls showed that patients were less accurate on all tasks with one exception: sv-PPA and controls had similar scores for the syntactic task (semantic task: lv-PPA vs. controls $F(1,32)=16.76$, $\mathrm{p}<0.001$, sv-PPA vs. controls $\mathrm{F}(1,30)=18.10, \mathrm{p}<0.001$; syntactic task: 1v-PPA vs. controls $\mathrm{F}(1,32)=7.71, \mathrm{p}=0.009$, sv-PPA vs. controls $\mathrm{F}(1,30)=2.06, \mathrm{p}=0.162$; word-form task: lv-PPA vs. controls $\mathrm{F}(1,32)=22.59, \mathrm{p}<0.001$, sv-PPA vs. controls $\mathrm{F}(1,30)=22.94, \mathrm{p}<0.001)$. Finally, we compared lv-PPA and sv-PPA. Sv-PPA patients had poorer performance than lv-PPA patients in the semantic task $(\mathrm{F}(1,18)=8.69, \mathrm{p}=0.046)$ whereas performance was similar in the word-form and in the syntactic task (both $\mathrm{Fs}<1$ ).

\subsection{Refined analyses and dissociations in the implicit processing tasks}


The results of the three explicit tasks allowed for subdividing the whole PPA group into six subgroups yielding for each lexical aspect a subgroup of 'good performers' (SEM+, SYNT+, FORM+) and of 'poor performers' (SEM-, SYNT-, FORM-). This approach aimed at refining the analyses on the three priming tasks to provide evidence for i) the existence of genuine priming effects in PPA, and ii) for multiple dissociations with respect to the semantic, syntactic and word-form components of the lexicon. We used accuracy scores in the different explicit tasks to disentangle good and poor performers in the three lexical domains. The SEM+, the SYNT+ and the FORM+ subgroups each contained the 10 best performers in the semantic, syntactic and word-form tasks, respectively. Conversely, the SEM-, the SYNT- and the FORM- subgroups contained the 10 poorest performers in the semantic, syntactic and word-form tasks, respectively.

ANOVAs were conducted by participants (F1) and by items (F2) for each of the six subgroups. The dependent variable was RT and the independent variable was "target type" (related, unrelated). For each participant, incorrect responses and missing data were excluded from the analyses.

In the semantic priming task the SEM+ subgroup had faster RT for related targets $(955 \mathrm{~ms} \pm$ 253) than for unrelated targets $(1046 \mathrm{~ms} \pm 254 ;(\mathrm{F} 1(1,9)=8,57, \mathrm{p}=0,017 ; \mathrm{F} 2(1,58)=6,79$, $\mathrm{p}=0,012$ ) corresponding to a significant priming effect of $91 \mathrm{~ms}$. In contrast, the SEMsubgroup had similar RT for related targets $(1309 \mathrm{~ms} \pm 451)$ and for unrelated targets (1266 $\mathrm{ms} \pm 465 ;(\mathrm{F} 1(1,9)=2,75, \mathrm{p}=0,131 ; \mathrm{F} 2(1,58)=1,08, \mathrm{p}=0,304)$. The comparison of overall $\mathrm{RT}$ in the SEM+ and SEM- subgroups showed that they were comparable in that they had similar RT $(\mathrm{F} 1(1,18)=2.99, \mathrm{p}=0.11 ; \mathrm{F} 2<1)$. In the syntactic priming task the SYNT+ subgroup had faster RT for related targets $(968 \mathrm{~ms} \pm 336)$ than for unrelated targets $(1032 \mathrm{~ms} \pm 387$; $\mathrm{F} 1(1,9)=6,82, \mathrm{p}=0,028 ; \mathrm{F} 2(1,58)=6,66, \mathrm{p}=0,029)$, corresponding to a significant priming effect of $64 \mathrm{~ms}$. In contrast, the SYNT- subgroup had similar RT for related targets (1261 ms 
$\pm 415)$ and for unrelated targets (1237 $\mathrm{ms} \pm 418$; both Fs $<1)$. The comparison of overall RT in the SYNT+ and SYNT- subgroups showed that they were comparable in that they had similar $\mathrm{RT}(\mathrm{F} 1(1,18)=1.41, \mathrm{p}=0.25 ; \mathrm{F} 2<1)$. In the word-form priming task the FORM+ subgroup had faster RT for related targets $(1228 \mathrm{~ms} \pm 294)$ than for unrelated targets $(1323 \mathrm{~ms} \pm 336$; $\mathrm{F} 1(1,9)=36,64, \mathrm{p}<0,001 ; \mathrm{F} 2(1,58)=5,09, \mathrm{p}=0,028)$, corresponding to a significant priming effect of 95 ms. In contrast, the FORM- subgroup had similar RT for related targets (1219 ms $\pm 344)$ and for unrelated targets (1184 $\mathrm{ms} \pm 387$; both Fs $<1)$. The comparison of overall RT in the FORM+ and FORM- subgroups showed that they were comparable in that they had similar RT (both Fs<1).

In addition, the following dissociations were found: 1) the SEM+ subgroup which had significant priming effects in the semantic task did not have any significant priming effects in the syntactic or the word-form task (syntactic task: related targets $1014 \mathrm{~ms} \pm 287$, unrelated targets $987 \mathrm{~ms} \pm 274$; both $\mathrm{Fs}<1$. Word-form task: related targets $1120 \mathrm{~ms} \pm 268$, unrelated targets $1157 \mathrm{~ms} \pm 315 ; \mathrm{F} 1(1,9)=1.36, \mathrm{p}=0.273, \mathrm{~F} 2(1,58)=1.44, \mathrm{p}=0.235) .2)$ The SYNT+ subgroup which had significant priming effects in the syntactic task did not have any significant priming effects in the semantic or the word-form task (semantic task: related targets $1048 \mathrm{~ms} \pm 358$, unrelated targets $1087 \mathrm{~ms} \pm 324 ; \mathrm{F} 1(1,9)=1.83, \mathrm{p}=0.209, \mathrm{~F} 2<1$. Wordform task: related targets $1124 \mathrm{~ms} \pm 310$, unrelated targets $1142 \mathrm{~ms} \pm 358 ; \mathrm{F} 1<1$, $\mathrm{F} 2(1,58)=1.02, \mathrm{p}=0.317) .3)$ The FORM + subgroup which had significant priming effects in the word-form task did not have any significant priming effects in the semantic or the syntactic task (semantic task: related targets $1189 \mathrm{~ms} \pm 427$, unrelated targets $1239 \mathrm{~ms} \pm 429$; $\mathrm{F} 1(1,9)=1.27, \mathrm{p}=0.289, \mathrm{~F} 2<1$. Syntactic task: related targets $1203 \mathrm{~ms} \pm 437$, unrelated targets $1185 \mathrm{~ms} \pm 413$; both $\mathrm{Fs}<1$ ).

In summary, we found multiple dissociations between the three lexical components: 1) Priming for lexical semantics but not for lexical syntax (SEM+ subgroup), 2) Priming for 
lexical syntax but not for lexical semantics (SYNT+ subgroup), 3) Priming for word-form representations but not for lexical semantics (FORM+ subgroup), 4) Priming for lexical semantics but not for word-form representations (SEM+ subgroup), 5) Priming for lexical syntax but not for word-form representations (SYNT+ subgroup), 6) Priming for word-form representations but not for lexical syntax (FORM+ subgroup). The priming dissociations revealed by the different PPA subgroups are illustrated in Table 3.

Moreover, such dissociations were also present at the individual level in several patients. We identified three patients demonstrating normal and significant priming effects as compared to priming in controls in the semantic task $(\mathrm{p}>0.1)$ but not any priming effect in the syntactic or the word-form task $(\mathrm{p}<0.05)$. Three other patients demonstrated normal and significant priming as compared to controls in the word-form task $(\mathrm{p}>0.1)$ but not in the syntactic or the semantic task $(p<0.05)$. Finally, one patient had normal and significant priming effects as compared to controls in the syntactic task $(\mathrm{p}>0.1)$ but not in the semantic or the word-form task (both $\mathrm{p}<0.05$ ).

Table 3 Multiple dissociations of priming effects in the SEM+, SYNT+ and FORM+ subgroups of PPA.

\begin{tabular}{lccc}
\hline & Semantic priming & Syntactic priming & Word-form priming \\
\hline SEM+ subgroup & $91 \mathrm{~ms} *$ & $-27 \mathrm{~ms}(\mathrm{~ns})$ & $37 \mathrm{~ms}(\mathrm{~ns})$ \\
SYNT+ subgroup & $39 \mathrm{~ms}(\mathrm{~ns})$ & $64 \mathrm{~ms} *$ & $18 \mathrm{~ms}(\mathrm{~ns})$ \\
FORM+ subgroup & $50 \mathrm{~ms}(\mathrm{~ns})$ & $-18 \mathrm{~ms}(\mathrm{~ns})$ & $95 \mathrm{~ms} *$ \\
\hline
\end{tabular}

$*=$ significant at $\mathrm{p}<0.05 . \mathrm{ns}=$ not significant. 


\subsection{Cortical thickness analyses}

The whole-brain between-group analysis showed lower cortical thickness in PPA patients as compared to controls in the left lateral temporal cortex. In sv-PPA, cortical thickness was diminished primarily in the left anterior and middle lateral temporal cortex, and in lv-PPA thickness was diminished essentially in the left middle temporal cortex and in posterior regions of the middle and inferior temporal gyrus. In lv-PPA and sv-PPA regions of reduced cortical thickness had a non negligible overlap and the whole PPA group demonstrated reduced cortical thickness of almost all regions of lateral temporal cortices. Results are illustrated in Figure 4.

\section{Discussion}

We explored the internal organization of the lexicon assessing lexical-semantic, syntacticgender and word-form aspects in a relatively large cohort of PPA patients to show multiple behavioral dissociations using comparable experimental paradigms. We also sought to confirm the central role of temporal cortices in the implementation of the three lexical components and to characterize their potential differential breakdown in lv-PPA and sv-PPA. Our main findings provide clear evidence for the existence of distinct syntactic, semantic and word-form components, thus demonstrating a threefold segregation of the mental lexicon. The use of a combined lv/sv-PPA model furthermore suggests the anatomical implementation of these components by lateral temporal cortices.

Results with healthy adults showed significant semantic, syntactic and word-form priming effects indicating that the three different types of word information are stored in the lexicon and that the corresponding lexical representations are sensitive to spreading pre-activation. 
Patient data from the priming tasks suggested that the different lexical representations are degraded in both PPA variants, but the explicit processing tasks demonstrated that this impairment is distinct in sv-PPA and lv-PPA. Furthermore, the sub-division of patients according to semantic, syntactic and word-form performance on the three explicit tasks showed that good performers in a given lexical domain demonstrated significant effects in the corresponding priming task whereas poor performers did not. Moreover, the subgroups having significant priming in a given domain did not display significant priming in the two other lexical domains. These results suggest that priming effects exist in subgroups of PPA patients, that their absence reflects damage to the tested lexical component and that the three components are functionally segregated as shown by multiple dissociations at the group level. The functional segregation of lexical components was also corroborated by priming data of several patients providing evidence for dissociations at the individual level. Finally, cortical thickness analyses revealed predominantly left-lateralized atrophy of the lateral temporal cortex, with lv-PPA patients demonstrating diminished thickness mainly in posterior-middle temporal cortices and sv-PPA having diminished thickness primarily in anterior and middle temporal regions, thus presenting non negligible overlap. The combined lv/sv-PPA cohort therefore provided a valuable model of damage to almost all regions of left lateral temporal cortices. This result indicates that the semantic, syntactic-gender and word-form components of the lexicon involve the lateral temporal cortex and that the functional segregation might be linked to an anatomical segregation within this brain region.

\subsection{Functional segregation of the lexicon}

Several studies have suggested that the three lexical components can be dissociated at the behavioral level. However, they were mostly based on case reports (Badecker et al., 1989) or small-cohort studies (e.g., Biran and Fridmann, 2012; Caramazza and Miozzo, 1997; Kavé 
and Lévy, 2003), and they did not compare the three components in a direct way, thus limiting the generalization of their results towards a comprehensive data-driven model of the lexicon. In the current study we provided evidence for multiple dissociations at the group level using a relatively large cohort of PPA patients and implementing directly comparable tasks. One might ask whether non-lexical factors, such as attention disorders in PPA, could have abolished priming effects in the whole group given that reaction times were significantly slower in PPA than in controls. However, refined analyses after splitting the whole group in good and poor lexical performers on the basis of an independent explicit task show that this is not the case. Significant priming dissociations at both the group and the individual patient level demonstrate that PPA patients are sensitive to priming, and that the differential sensitivity resulting in dissociations is not due to task-related effects but to genuine lexical deficits. Thus, the segregation of the lexicon into three components is supported by our cohort data which substantiates previous single-case and small-cohort studies which only revealed “one vs. one component" dissociations such as lexical syntax vs. word-form representations (Caramazza and Miozzo, 1997), or lexical-syntax vs. lexical-semantic representations (Biran and Fridmann, 2012; Kavé and Lévy, 2003).

The existence of distinct lexical representations is also supported by our results with healthy adults demonstrating significant priming effects in the three distinct lexical domains. These findings replicate some results from previous studies using semantic and word-form priming paradigms (e.g., Pastizzo and Feldman, 2009; Velan and Frost, 2011), and they add new evidence with respect to syntactic priming in healthy adults, which is still debated. Gender priming effects have been demonstrated by Akhutina et al. (2001), whereas other authors did not find syntactic priming (e.g., Finocchiaro and Caramazza, 2006). Such diverging results might be linked to differences in task demands. Our data deriving from methodologically comparative assessments in healthy adults in the three lexical domains corroborate the 
existence of priming-sensitive semantic and word-form representations, and provide novel evidence for lexical-syntactic gender representations.

\subsection{Anatomical implementation of lexical components}

We also provide some hints with respect to the neural substrate of the lexical components. Previous imaging studies with healthy adults (Graves et al., 2008; Lau et al., 2013; Snijders et al., 2009) could not provide the anatomical implementation of the three components, mainly because they have not been compared in a direct way. The variability of hardly comparable tasks has generated some controversy with respect to a potential core region for lexical processing, suggesting that temporal, parietal and/or frontal regions might be involved. However, most studies appear to converge towards a crucial implication of lateral temporal cortices in the processing or storage of lexical information (Lau et al., 2013; Pillay et al., 2014; Schwartz et al., 2009; Tyler et al., 2011). We therefore used a lesion model of lateral temporal cortices, a merged lv/sv-PPA cohort, to check whether this region genuinely plays a central role in the implementation of lexical information and whether differential damage to this region results in functional dissociations of semantic, syntactic and word-form components. Our findings show that the PPA cohort demonstrates largely selective thinning of nearly the entire lateral temporal cortex, predominating on the left hemisphere. The priming data showing multiple intra-lexical dissociations and differential breakdown of the three lexical components in patients with differential damage to lateral temporal cortices therefore indicate that this cortical region plays a necessary core role in the processing of the different lexical aspects. In line with these findings, we propose that the lexicon is primarily implemented by the lateral temporal cortex and that the functional segregation of the three lexical components might correspond to an anatomical segregation within lateral temporal cortices. It should be noted that the evidenced behavioral-lexical dissociations were found in the combined lv/sv-PPA cohort-model and that they were not linked to the PPA variants as 
such. Lv-PPA and sv-PPA had different cortical thickness distributions but there was overlap of thickness reduction and large portions of lateral temporal cortices might be dysfunctional in both PPA variants. Thus the observed dissociations of the three lexical components are not linked to the two PPA variants per se but rather to subgroups of patients within the merged lv/sv-model. One crucial issue is now to identify the exact temporal-cortical correlates of the three lexical components. We conducted anatomo-functional correlation analyses but the merged PPA group (17 MRI scans), and even the combined PPA plus control group, did not provide sufficient statistical power to robustly identify the lexical correlates. Further studies with larger patient cohorts demonstrating damage to the temporal cortex are therefore required to carry out informative correlation analyses and to identify the cortical regions hosting each lexical component.

\subsection{Implications for PPA}

Our data also refine the understanding of lexical deficits in PPA. Previous studies have suggested that PPA patients have lexical disorders such as impaired lexical/verbal semantics in sv-PPA (Mesulam et al., 2013; 2014; Wilson et al., 2014) or lexical word-form deficits in lv-PPA (e.g., Leyton et al., 2012; Migliaccio et al., 2016; Teichmann et al., 2013). One should note that these studies did not use lexicon-specific tasks like verbal semantic priming or masked word-form priming as in the present investigation. In contrast to these two PPA variants, previous studies also suggest that agrammatic/nonfluent PPA demonstrates no or only slight lexical dysfunction as shown for example in naming tasks (Gorno-Tempini et al., 2004; Leyton et al., 2011; Migliaccio et al., 2016; Race et al., 2013). However, little is known about the exact damage to the different components of the lexicon in PPA. Our data from the explicit processing tasks indicate that sv-PPA patients have significant damage predominantly within both the semantic and the word-form component of the lexicon. In contrast, syntactic 
representations are relatively spared in both PPA variants. They also show that the lexical deficit in lv-PPA predominates on the word-form component of the lexicon with relatively spared lexical-semantic and syntactic representations. However, our priming data are more sensitive showing impairment of all three lexical components in lv-PPA and sv-PPA. Priming tasks thus might allow for identifying even slight lexical damage in PPA which could be important to guide specific rehabilitation strategies.

Our data also reinforce some findings suggesting that the linguistic deficit in the different PPA variants is more wide-spread than proposed in the international diagnostic criteria of Gorno-Tempini et al. (2011). Several studies have shown that word-finding deficits in lv-PPA are not only related to "lexical" access but that they are also caused by genuine semantic impairments, especially after some years of disease evolution (Leyton et al., 2013; Teichmann et al., 2013; Wicklund et al., 2014). Similarly, studies using lexical decision tasks on written words in sv-PPA seem to suggest that the orthographical word-form component of the lexicon could be impaired (Coltheart et al., 2010; Pulvermüller et al., 2010; Rogers et al., 2004). Our data appear to confirm this latter assumption via the use of a masked priming task tapping orthographical word-forms while minimizing potential semantic biases due to the masked procedure. Generally speaking, our results strengthen previous results through the use of wellcontrolled priming tasks, and indicate that lv-PPA and sv-PPA progressively erode the entire range of lexical representations by damaging their respective cortical underpinnings. Such erosion might evolve along a gradient related to disease evolution and to progressive damagespreading within the lateral temporal cortex.

\section{Conclusion}

Our data strongly support language models of three separate lexical components (e.g., Carramaza and Miozzo, 1997) and suggest that the semantic/syntactic/word-form segregation 
is implemented by lateral temporal cortices. They also provide the basis for larger patient cohort or functional imaging studies to specify the temporal-cortical underpinnings of each component. With respect to PPA, our results generate detailed insight into lexical dysfunction, which could have an impact on diagnosis and rehabilitation strategies.

\section{Acknowledgements}

This work was supported by funding from the program "Investissements d'avenir" ANR-10IAIHU-06 (Agence Nationale de la Recherche-10-Investissements-Avenir-Institut HospitaloUniversitaire-06). 


\section{References}

Akhutina, T., Kurganski, A., Kurganskaya, M., Polinski, M., Polonskaya, N., Larina, O., et al. (2001). Processing of grammatical gender in normal and aphasic speakers of Russian. Cortex, 37, 295-326.

Badecker, W., Miozzo, M., \& Caramazza, A. (1995). The two-stage model of lexical retrieval: evidence from a case of anomia with selective preservation of grammatical gender. Cognition, 57, 193-216.

Binder, J.R., McKiernan, K.A., Parsons, M.E., Westbury, C.F., Possing, E.T., Kaufman, J.N., et al. (2003). Neural correlates of lexical access during visual word recognition. Journal of Cognitive Neuroscience, 15, 372-93.

Biran, M., \& Friedmann, N. (2012). The representation of lexical-syntactic information: Evidence from syntactic and lexical retrieval impairments in aphasia. Cortex, 48, 1103-27.

Caramazza, A. (1997). How many levels of processing are there in lexical access? Cognitive Neuropsychology, 14, 177-208.

Caramazza, A., \& Miozzo, M. (1997). The relation between syntactic and phonological knowledge in lexical access: evidence from the tip-of-the-tongue phenomenon. Cognition, $64,309-43$

Cardebat, D., Doyon, B., Puel, M., Goulet, P., \& Joanette, Y. (1990). Evocation lexicale formelle et sémantique chez des sujets normaux. Performances et dynamiques de la production en fonction du sexe, de l'âge et du niveau d'étude. Acta Neurologica Belgica, 90, 207-17.

Caviness, V.S., Makris, N., Montinaro, E., Sahin, N.T., Bates, J.F., Schwamm, L., et al. (2002). Anatomy of stroke, Part I: an MRI-based topographic and volumetric System of analysis. Stroke, 33, 2549-56. 
Coltheart, M., Saunders, S.J., \& Tree, J.J. (2010). Computational modelling of the effects of semantic dementia on visual word recognition. Cognitive Neuropsychology, 27, 101-14.

Deloche, G., \& Hannequin, D. (1997). Test de dénomination orale d'images : DO 80. Paris: Éditions du Centre de Psychologie Appliquée.

Devlin, J.T., Russell, R.P., Davis, M.H., Price, C.J., Wilson, J., Moss, H.E., et al. (2000). Susceptibility-induced loss of signal: comparing PET and fMRI on a semantic task. Neuroimage, 11, 589-600.

Dubois, B., Slachevsky, A., Litvan, I., \& Pillon, B. (2000). The FAB, a frontal assessment Battery at bedside. Neurology, 55, 1621-1626.

Finocchiaro, C., \& Caramazza, A. (2006). The production of pronominal clitics: Implications for theories of lexical access. Language and Cognitive Processes, 21, 141-80.

Fischl, B., \& Dale, A.M. (2000). Measuring the thickness of the human cerebral cortex from magnetic resonance images. Proceedings of the National Academy of Sciences of the United States of America, 97, 11050-55.

Folstein, M., Folstein, S., \& McHugh, P.R. (1975). Mini-Mental State: a practical method for grading the cognitive state of patients for the clinician. Journal of Psychiatric Research, $12,189-198$.

Friederici, A., Ruschemeyer, S.A., Hahne, A., \& Fiebach, C.J. (2003). The role of left inferior frontal and superior temporal cortex in sentence comprehension: localising syntactic and semantic processes. Cerebral Cortex, 13(2), 70-7.

Garavan, H., Ross, T.J., Li, S.J., \& Stein, E.A. (2000). A parametric manipulation of central executive functioning. Cerebral Cortex, 10, 585-92.

Gorno-Tempini, M.L., Brambati, S.M., Ginex, V., Ogar, J., Dronkers, N.F., Marcone, A., et al. (2008). The logopenic/phonological variant of primary progressive aphasia. Neurology, $71,1227-34$ 
Gorno-Tempini, M.L., Dronkers, N., Ranklin, K.P., Ogar, J., Phengrasami, L., Rosen, H.J., et al. (2004). Cognition and Anatomy in three variants of Primary Progressive Aphasia. Annals of Neurology, 55, 335-46.

Gorno-Tempini, M.L., Hillis, A.E., Weintraub, S., Kertesz, A., Mendez, M., Cappa, S.F., et al. (2011). Classification of Primary Progressive Aphasia and its variants. Neurology, 76, 1006-14.

Graves, W.W., Desai, R., Humphries, C., Seidenberg, S.S., \& Binder, J.R. (2010). Neural systems for reading aloud: A multiparametric approach. Cerebral Cortex, 20, 1799-1815.

Graves, W.W., Grabowski, T.J., Mehta, S., \& Gupta, P. (2008). Left posterior superior temporal gyrus participates specifically in accessing lexical phonology. Journal of Cognitive Neuroscience, 20, 1698-1710.

Henaff Gonon, M.A., Bruckert, R., \& Michel, F. (1989). Lexicalization in an anomic patient. Neuropsychologia, 27, 391-407.

Jackendoff, R. (2002). Foundations of Language. (1 ${ }^{\text {st }}$ ed.). New York: Oxford University Press.

Kavé, G., \& Levy, Y. (2003). Sensitivity to gender, person and tense inflection by persons with Alzheimer's disease. Brain and Language, 87, 267-77.

Kuperberg, G., Lakshmanan, B., Greve, D., \& West, W. (2008). Task and semantic relationship influence both the polarity and localization of hemodynamic modulation during lexico-semantic processing. Human Brain Mapping, 29, 544-61.

Lau, E.F., Gramfort, A., Hamalainen, M.S., \& Kuperberg, G.R. (2013). Automatic semantic facilitation in anterior temporal cortex revealed through multimodal neuroimaging. Journal Neuroscience, 33, 17174-81. 
Lau, E.F., Weber, K., Gramfort, A., Hamalainen, M.S., \& Kuperberg, G.R. (2014). Spatiotemporal signatures of lexical-semantic prediction. Cerebral Cortex. http://dx.doi.org/10.1093/cercor/bhu219

Levelt, W. (1992). Accessing words in speaking production: stages, processes and representations. Cognition, 42, 1-22.

Leyton, C.E., Villemagne, V.L., Savage, S., Pike, K.E., Ballard, K.J., Piguet, O., et al. (2011). Subtypes of progressive aphasia: application of the International Consensus Criteria and validation using beta-amyloid imaging. Brain, 134, 3030-43.

Leyton, C. E., Piguet, O., Savage, S., Burrell, J., \& Hodges, J.R. (2012). The neural basis of logopenic progressive aphasia. Journal of Alzheimer's Disease, 32, 1051-1059.

Leyton, C.E., Hsieh, S., Mioshi, E., \& Hodges J.R. (2013). Cognitive decline in logopenic aphasia: more than losing words. Neurology, 80, 897-903.

Mazaux, J.M., \& Orgogozo, J.M. (1982). Boston Diagnostic Aphasia Examination. Adaptation française. Paris: Éditions du Centre de Psychologie Appliquée.

Mesulam, M.M., Wieneke, C., Hurley, R., Rademaker, A., Thompson, C.K., Weintraub, S., et al. (2013). Words and objects at the tip of the left temporal lobe in primary progressive aphasia. Brain, 136, 601-18.

Mesulam, M., Rogalski, E., Wieneke, C., Hurley, R., Geula, C., Bigio, E., et al. (2014). Primary Progressive Aphasia and evolving neurology of the language network. Nature Reviews Neurology, 10, 554-69.

Migliaccio, R., Boutet, C., Valabregue, R., Ferrieux, S., Nogues, M., Lehéricy, S., et al. (2016). The Brain Network of Naming: A Lesson from Primary Progressive Aphasia. PLoS One, 11(2), e0148707.

Neely, J.H. (1976). Semantic priming and retrieval from lexical memory: Evidence for facilitatory and inhibitory processes. Memory \& Cognition, 4(5), 648-654. 
New, B., Pallier, C., Ferrand, L., \& Matos, R. (2004). Une base de données lexicales du français contemporain sur internet: LEXIQUE 2. Année Psychologique, 101, 447-62.

Oldfield, R.C. (1996). Things, words and the brain. Quarterly Journal of Experimental Psychology, 18, 340-53.

Pallier, C., Devauchelle, A.D., Dehaene, S. (2011). Cortical representation of the constituent structure of sentences. Proceedings of the National Academy of Sciences of the United States of America,108(6), 2522-7.

Pastizzo, M.J., \& Feldman, L.B. (2009). Multiple dimensions of relatedness among words: conjoint effects of form and meaning on word recognition. Mental Lexicon, 41, 1-25.

Pillay, S.B., Stengel, B.C., Humphries, C., Book, D.S., \& Binder, J.R. (2014). Cerebral localization of impaired phonological retrieval during rhyme judgement. Annals of Neurology, 76, 738-46.

Pulvemuller, F., Cooper-Pye, E., Dine, C., Olaf Hauk, Nestor, P.J. \& Patterson, K. (2010). The word processing deficit in Semantic Dementia: all categories are equal, but some categories are more equal than others. Journal of Cognitive Neuroscience, 22, 2027-2041.

Race, D., Tsakpini, K., Crinion, J., Newhart, M., Davis, C., Gomez, Y., et al. (2013). An area essential for linking word meanings to word forms: Evidence from primary progressive aphasia. Brain and Language, 127, 167-76.

Rogalski, E., Cobia, D., Harrison, T.M., Wieneke, C., Thompson, C.K., Weintraub, S., et al. (2011). Anatomy of language impairments on Primary Progressive Aphasia. Journal of Neuroscience, 31, 3344-50.

Rogers, T.T., Lambon Ralph, M.A., Hodges, J.R., \& Patterson, K. (2004). Natural selection: The impact of semantic impairment on lexical and object decision. Cognitive Neuropsychology, 21, 331-352. 
Ruff, I., Blumstein, S.E., Myers, E.B., \& Hutchison, E. (2008). Recruitment of anterior and posterior structures in lexical semantic processing: an fMRI study comparing implicit and explicit tasks. Brain and Language, 105, 41-49.

Schwartz, M., Kimberg, D., Walker, G., Faseyitan, O., Brecher, A., Dell, G., et al. (2009). Anterior temporal involvement in semantic word retrieval: voxel-based lesion-symptom mapping evidence from aphasia. Brain, 132, 3411-27.

Snijders, T., Vosse, T., Kempen, G., Van Berkum, J., Petersson, K., \& Hagoort, P. (2009). Retrieval and unification of syntactic structure in sentence comprehension: an fMRI study using word-category ambiguity. Cerebral Cortex, 19, 1493-1503.

Spitsyna, G., Warren, J.E., Scott, S.K., Turkheimer, F.E., \& Wise, R.J. (2006). Converging language streams in the human temporal lobe. Journal of Neuroscience, 26, 7328-36.

Teichmann, M., Kas, A., Boutet, C., Ferrieux, S., Nogues, M., Samri, D., et al. (2013). Deciphering logopenic primary progressive aphasia: a clinical, imaging and biomarker investigation. Brain, 136, 3474-88.

Tyler, L., Marslen-Wilson, W., Randall, B., Wright, P., Devereux, B., Zhuang, J., et al. (2011). Left inferior frontal cortex and syntax: function, structure and behavior in patients with left hemisphere damage. Brain, 134, 415-31.

Velan, H., \& Frost, M. (2011). Words with and without internal structure: what determines the nature of orthographic and morphological processing? Cognition, 118, 141-56.

Wicklund, M.R., Duffy, J.R., Strand, E.A., Machulda, M.M., Whitwell, J.L., Josephs, K.A. (2014). Quantitative application of the primary progressive aphasia consensus criteria. Neurology, 82, 1119-26.

Wilson, S.M., DeMarco, A.T., Henry, M.L., Gesierich, B., Babiak, M., Mandelli, M.L., et al. (2014). What role does the anterior temporal lobe play in sentence-level processing? 
Neural correlates of syntactic processing in semantic PPA. Journal of Cognitive Neuroscience, 26, 970-85.

Wise, R.J. (2003). Language systems in normal and aphasic human subjects: functional imaging studies and inferences from animal studies. British Medical Bulletin, 65, 95-119.

Worsley, K.J., Andermann, M., Koulis, T., MacDonald, D., \& Evans, A.C. (1999). Detecting changes in nonisotropic images. Human Brain Mapping, 8, 98-101. 


\section{Figures}

Figure 1 Illustration of the stimuli and time course of the three implicit processing tasks (A). First line: semantic priming (honey-bee; cardboard-bee); second line: syntactic priming (concordant gender determinant-flour; non concordant gender determinant-flour); third line: word-form priming (e.g., beer-DEER; e.g., foot-DEER). Illustration of the stimuli for the three explicit processing tasks (B). First column: semantic task (piano; flower vs. violin); second column: syntactic gender task (piano; concordant determiner vs. non-concordant determiner); third column: word-form task (pian_; 'a' vs. 'o').

A
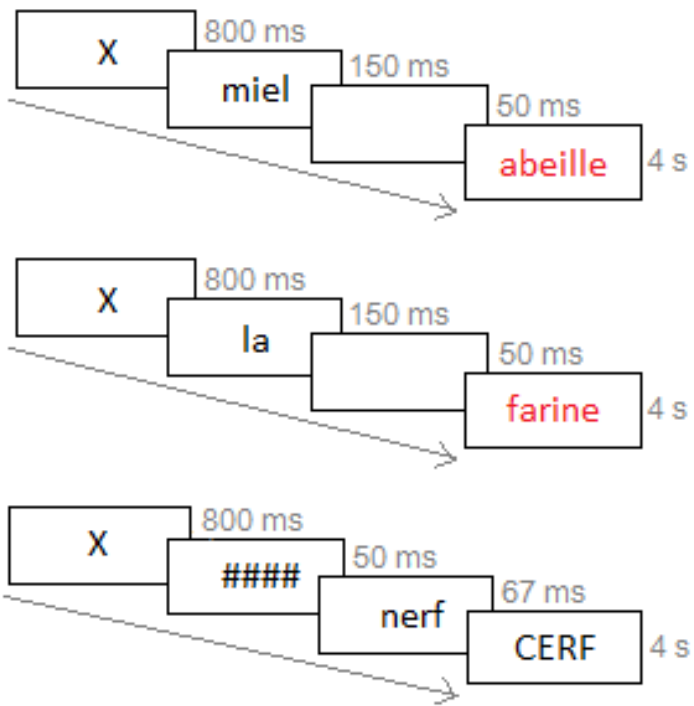
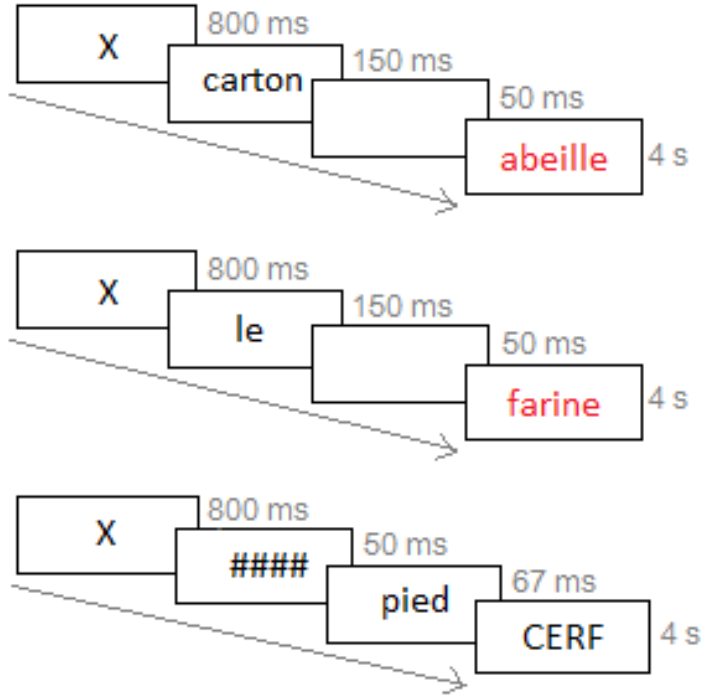

B

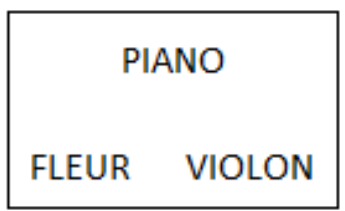

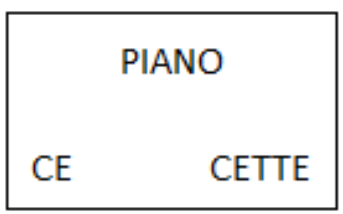

PIAN_

음 
Figure 2 Priming effects as reflected by the comparison between unrelated and related targets in the semantic (A), syntactic (B) and word-form (C) priming tasks for healthy controls, the whole PPA group, lv-PPA and sv-PPA $(*=\mathrm{p}<0.05)$.

A

\section{Semantic priming task}

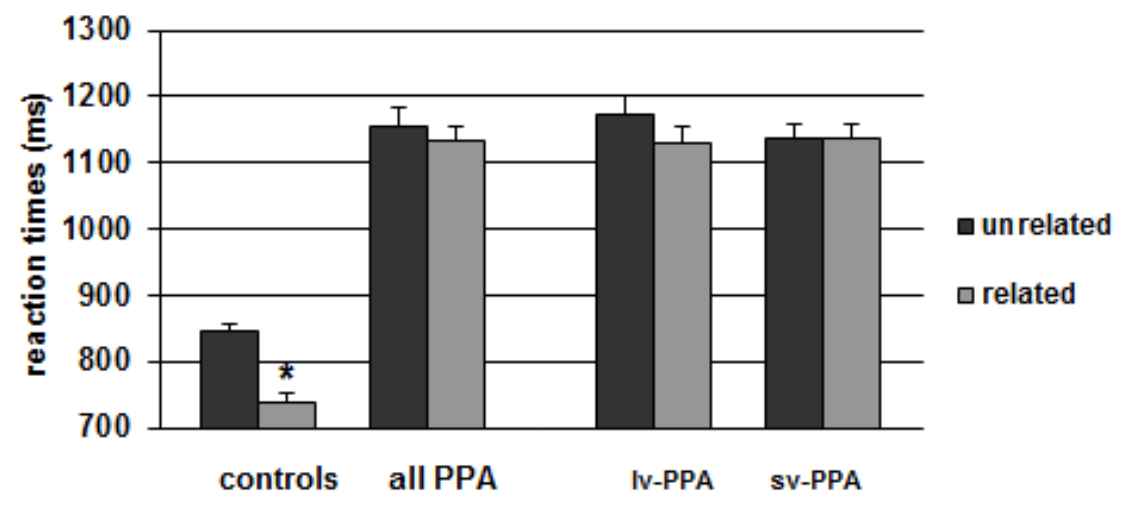

B

\section{Syntactic priming task}

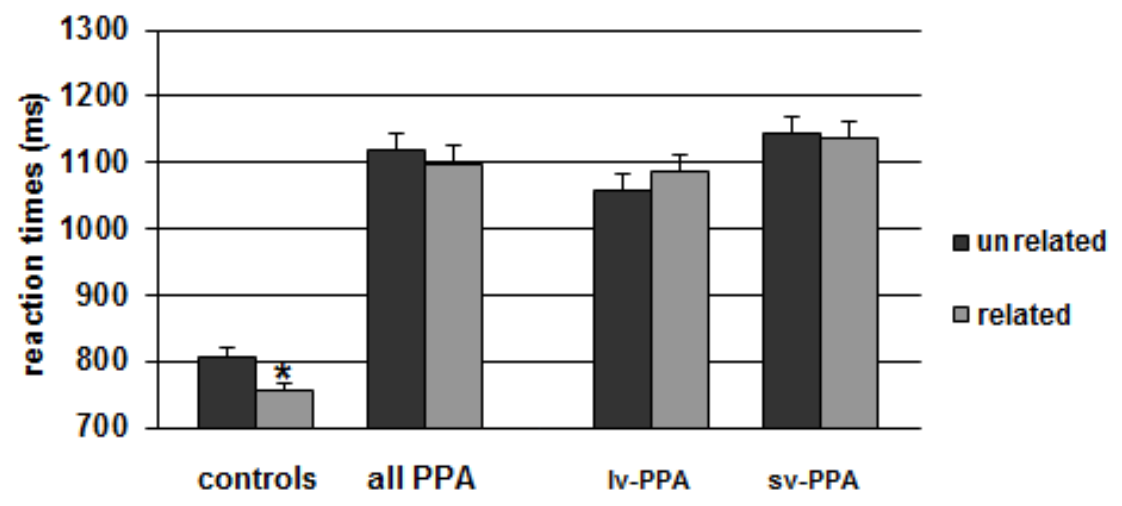

c

\section{Word-form priming task}

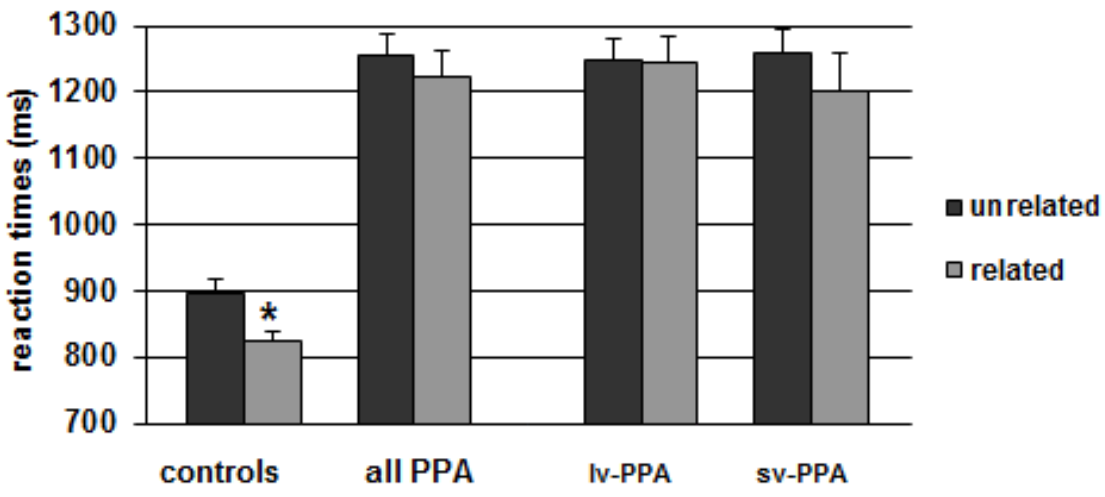


Figure 3 Performance on the three explicit processing tasks for healthy controls, lv-PPA and $\operatorname{sv-PPA}(*=p<0.05)$

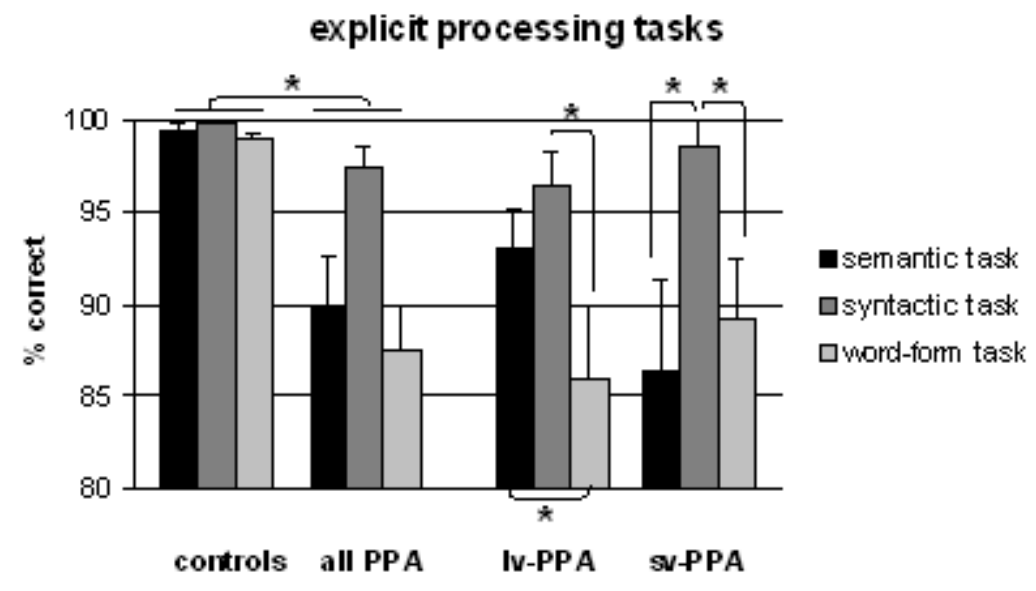


Figure 4 Regions of significantly reduced cortical thickness in PPA patients versus healthy controls. Correction for multiple comparisons using the Random Field Theory, with corrected p-values: $\mathrm{p}<0.05$ for clusters. A) whole PPA group, B) lv-PPA, C) sv-PPA. The color bar represents p-values varying between " 0.05 " and " 0 ". Note that the zero p-values are not strictly " 0 " but that they are close to "0".
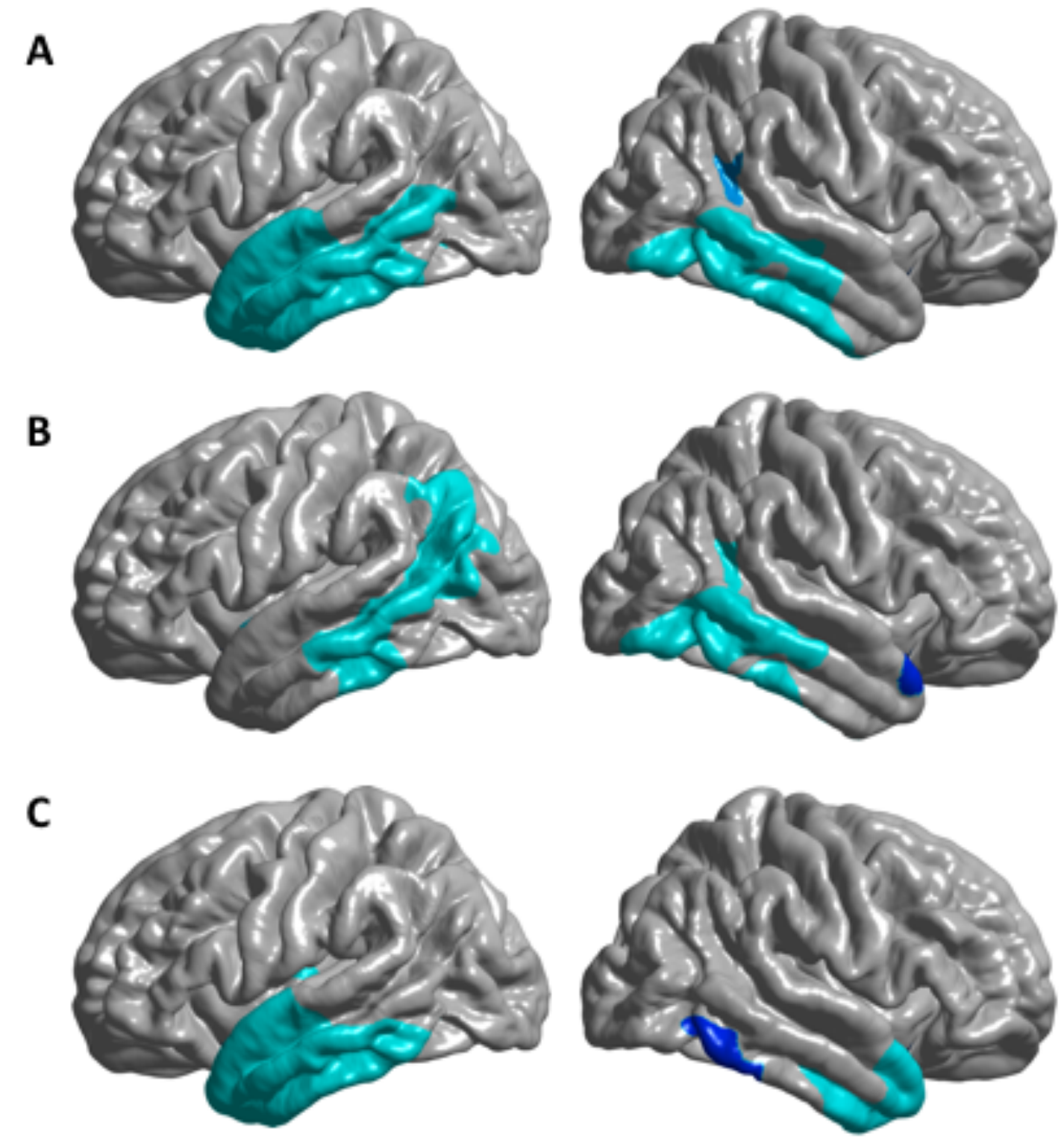

Left hemisphere

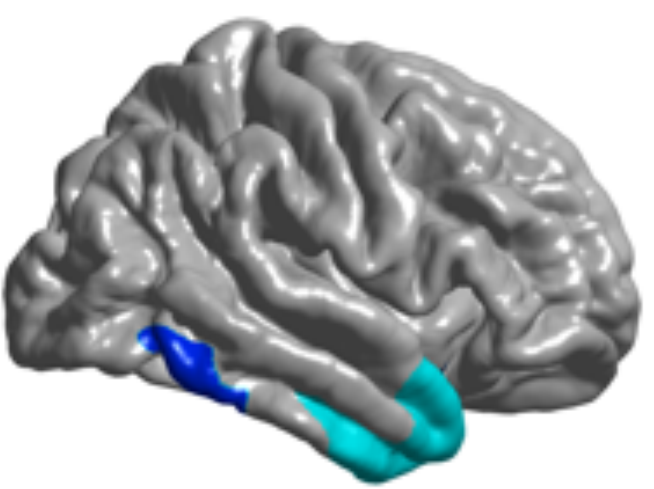

Right hemisphere

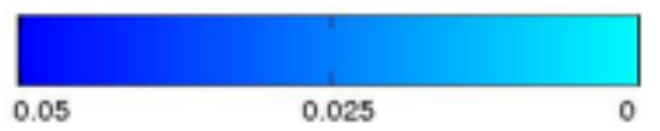

